\title{
LA SEPARABILIDAD DE LA MENTE
}

\author{
Francisco Rodríguez Valls \\ Universidad de Sevilla
}

\begin{abstract}
Resumen: Este ensayo trata sobre los argumentos que pueden esgrimirse a favor de la separabilidad de la mente. La finalidad es comprobar si es posible transferir la mente humana a un cuerpo sintético o bien no es posible. Si la mente es separable del cuerpo humano, no necesita otro para sobrevivir. Si no lo es, el problema queda reducido a hallar las técnicas que permitan la transferencia.

Palabras clave: separabilidad, mente, inteligencia artificial, transferencia.

Separability of Mind
\end{abstract}

Abstract: This paper deals with the reasons can be argued in order to demonstrate the separability of mind. Its goal is to check if it is possible to transfer human mind to a synthetic body or not. If mind is separable from human body, it is not necessary for it another body to survive. If not, the problem is just the one of finding technical tools to allow the transference.

Keywords: separability, mind, artificial intelligence, transference.

Recibido: 31/08/2018 Aprobado: 20/02/2019

\section{Planteamiento del problema}

Creo que nadie se escandalizará si afirmo que el ser humano es un complejo biopsíquico que habitualmente nace y se desarrolla en un entorno socio-cultural. Eso es demasiado obvio y, sin embargo, ahí se encuentran los tres términos del problema que quiero tratar, las condiciones de posibilidad de intelección de la complejidad del asunto: un cuerpo vivo, unas estructuras psicológicas determinadas y un entorno en el que se aprenden los valores con los que construir y estructurar la existencia.

En primer lugar, un cuerpo vivo. Los elementos del organismo corporal no nos sirven si no funcionan. Es al cuerpo vivo al que tenemos que prestar atención. Un cuerpo "psíquico" en cuanto está insuflado de aliento, que es la raíz etimológica de psique. 
En segundo lugar, unas estructuras psicológicas bien determinadas que diferencian lo humano del resto de las especies animales. Existen múltiples formas de psiquismos que interaccionan con el medio. El intelectivo es uno de ellos. Y esto sin entrar en la discusión de si se ha producido algún salto esencial del resto de especies animales al ser humano o bien puede hablarse de un gradualismo que aporta facultades nuevas pero que puede ser resultado de los mecanismos evolutivos por la supervivencia de la especie en las mejores condiciones posibles.

En tercer lugar, existe lo que podríamos llamar con el nombre de "útero cultural": un metafórico receptáculo en el que el sujeto recibe la formación de manera inconsciente en sus primeros años de vida y que será esencial en el desarrollo de sus costumbres y creencias a lo largo de toda su existencia natural.

Estos tres elementos entran en interacción en el ser humano de manera evidente, de forma que lo primero que apreciamos de él es que consiste en una unidad bio-psíquico-cultural: es un cuerpo vivo endoculturado. Y esta primera apreciación, afirmaba al principio, parece que no debe suscitar graves problemas teóricos.

Pero el saber es enemigo de lo obvio y una de las maneras que tiene el conocimiento de operar es el análisis de la realidad para intentar comprenderla mejor. En este sentido podemos encontrar dos posturas filosóficas contrapuestas que responden a intereses de épocas diferentes: la racionalista y la fenomenológica. La racionalista aboga por la descomposición hasta las últimas piezas del lego, como dejó bien claro Descartes en la segunda parte del Discurso del método y Spinoza en el Tratado para la reforma del entendimiento. La fenomenológica insiste en que para comprender el fenómeno no hay que analizarlo sino percibir el sentido que tiene en su completo darse: el objeto de conocimiento de lo humano es la vivencia que el sujeto posee. Precisamente por ello, por poner un ejemplo, el fenomenólogo de la existencia Karl Jaspers afirma en su Psicopatología General que ir más allá de la unidad de sentido del sujeto humano como un todo a lo único que aboca 
es a problemas insalvables. Reconocemos que existen varios problemas irresolubles hasta el momento si queremos identificar los diferentes aspectos en sus formas "puras" y "separadas"; parece que Jaspers podría tener razón pues llevamos miles de años discutiendo sobre el dualismo sin aportar soluciones del todo satisfactorias. Hemos esbozado monismos de todo tipo para reducir los diferentes elementos a uno: materialistas, espiritualistas o neutrales, pero más que aportar soluciones a los problemas fundamentales despiertan otros aún de mayor gravedad.

Es evidente también que el cuerpo influye en la psique y que la psique tiene una base cerebral o, al menos, una importante relación con el cerebro. Un dolor fuerte debilita la capacidad de concentración. De la misma manera, se sabe desde antiguo que beber una cantidad importante de alcohol afecta a los niveles de conciencia y de control de la conducta; que un golpe en la cabeza puede hacer perder la consciencia, etc. Hasta tal punto sabemos eso hoy que se han desarrollado principios activos para controlar los estados de conciencia en los que el sujeto, especialmente el enfermo, se mueve. Se ha avanzado mucho en la farmacología de la sedación.

También sabemos que un estado psíquico emocional puede influir en la salud corporal. Las escuelas de medicina psicosomática han desarrollado con minuciosidad estas cuestiones y se han encontrado en la medicina clínica correlaciones entre problemas de estrés y enfermedades del corazón, del aparato digestivo o de la piel.

El tercer elemento, el cultural, parece que se ha trabajado menos en el siglo XX y está empezando a considerarse en el siglo XXI: pueden existir culturas enfermas porque están fundadas en valores que producen enfermedad en los ciudadanos. Eso ocurre en las sociedades de la competencia, esas a las que Han ha denominado "sociedades del cansancio" y Arana "sociedades del desencanto". No hay que extrañarse entonces que la riqueza y la opulencia de Occidente no vayan juntas con altas dosis de felicidad sino más bien de frustración, o que la satisfacción de las necesidades orgánicas y la dicha no vayan de la mano. 
$\mathrm{Y}$, sin embargo, "se mueve". El ser humano funciona como una unidad pero la filosofía quiere saber más de ello. No es una momia, no es un ángel, no es un puro valor cultural. Es una unidad, sí, pero de ahí surge una cuestión que ha interesado a los filósofos desde siempre y es la cuestión de si la muerte del cuerpo implica también la descomposición de la mente, del alma intelectiva, como también se descomponen las culturas, o puede hablarse de una separabilidad de la mente respecto de sus condiciones corporales y culturales. El cuerpo desaparece, la cultura también, ¿̇es la mente una excepción?

\section{Aristóteles y la función y separabilidad de la mente}

El primer autor que habla de la unidad humana de alma y cuerpo como de una unidad substancial y que la justifica con altas dosis de teoría es, al menos que yo conozca, Aristóteles. Los textos en los que se refiere al tema que nos ocupa se encuentran en Sobre el alma III, 4. Voy a citar dos, uno que habla de la naturaleza del conocimiento intelectual y otro que trata de forma directa de la separabilidad de la mente. En ellos se plantea de manera tentativa la posibilidad de la unidad, por una parte, y de la separabilidad, por otra. Es difícil conciliar ambas cosas, pero veamos cómo lo expone el Filósofo.

Respecto de la naturaleza del conocimiento intelectual, tema preliminar pero necesariamente relacionado con nuestra cuestión, dice el de Estagira lo siguiente:

Por lo que se refiere a aquella parte del alma con que el alma conoce y piensa [...] ha de examinarse cuál es su característica diferencial y cómo se lleva a cabo la actividad de inteligir. Ahora bien, si el inteligir constituye una operación semejante a la sensación, consistirá en padecer cierto influjo bajo la acción de lo inteligible o bien en algún otro proceso similar. Por consiguiente, el intelecto

— siendo impasible— ha de ser capaz de recibir la forma, es decir, ha de ser en 
potencia tal como la forma pero sin ser ella misma y será respecto de lo inteligible algo análogo a lo que es la facultad sensitiva respecto de lo sensible. Por consiguiente y puesto que intelige todas las cosas, necesariamente ha de ser sin mezcla — como dice Anaxágoras- para que pueda dominar o, lo que es lo mismo, conocer, ya que lo que exhibe su propia forma obstaculiza e interfiere a la ajena. Luego no tiene naturaleza alguna propia aparte de su misma potencialidad (429a 10-22).

Este texto es fundamental para establecer la naturaleza del realismo aristotélico: el intelecto no tiene naturaleza alguna propia. Si la tuviera, su consistencia obstaculizaría la intelección de las cosas tal y como son en sí mismas. El ejemplo de la tablilla encerada sobre la que deja su marca el sello es bien conocido y expresa bien la función que desea enunciar en el conocimiento sensible. Pero, ¿qué pasaría si en lugar de una tablilla encerada ponemos una base sólida de hierro y quitamos la cera? ¿Podría el sello dejar su huella? Lo tendría bastante más difícil. Si la deja, si algo de lo real conocemos, es porque la naturaleza de esa potencia o facultad debe tener la menor consistencia posible. Pero la pregunta que surge es la siguiente: ¿es esa consistencia tan mínima como para no tener ninguna? Evidentemente, la potencia sensitiva, los sentidos, tienen sus límites y sus umbrales, tienen una cierta naturaleza propia. ¿Pero la tiene la mente? ¿Puede captar las ideas de las cosas tal y como son en sí? ¿Sería la mente pura espiritualidad que no necesitaría estar incardinada en un cuerpo para conocer? Y es aquí donde el segundo texto que dije que iba a traer a colación adquiere su sentido. Dice así:

De ahí que sería igualmente ilógico que estuviera mezclado con el cuerpo: y es que en tal caso poseería alguna cualidad, sería frío o caliente y tendría un órgano como lo tiene la facultad sensitiva; pero no lo tiene realmente. Por lo tanto, dicen bien los que dicen que el alma es el lugar de las formas, si exceptuamos que no lo es toda ella, sino solo la intelectiva y que no es las formas en acto sino en potencia. Por lo demás y si se tiene en cuenta el funcionamiento de los órganos sensoriales y del sentido, resulta evidente que la impasibilidad de la 
facultad sensitiva y de la facultad intelectiva no son del mismo tipo: el sentido, desde luego, no es capaz de percibir tras haber sido afectado por un objeto fuertemente sensible, por ejemplo, no percibe el sonido después de sonidos intensos, ni es capaz de ver u oler, tras haber sido afectado por colores u olores fuertes; el intelecto, por el contrario, tras haber inteligido un objeto fuertemente inteligible, no intelige menos sino más, incluso, los objetos de rango inferior. $Y$ es que la facultad sensible no se da sin el cuerpo, mientras que el intelecto es separable. Y cuando éste ha llegado a ser cada uno de sus objetos a la manera en que se ha dicho que lo es el sabio en acto - lo que sucede cuando es capaz de actualizarse por sí mismo-, incluso entonces se encuentra en cierto modo en potencia, si bien no del mismo modo que antes de haber aprendido o investigado: el intelecto es capaz también entonces de inteligirse a sí mismo (429a 25-429b 9).

La mente es ajena a las categorías materiales: ¿cuánto pesa la mente?, ¿de qué color es?, ¿̇es dura o blanda?, ¿̇es grande o pequeña?, ¿̇es dulce o salada?, ¿es fría o caliente? Eso va indicando que sus diferencias con la naturaleza de la corporalidad son evidentes. ¿Pero lo son tanto como para decir, como explicita Aristóteles, que no tiene realmente un órgano como sí lo tiene cada facultad sensitiva? ¿No han venido a decir las neurociencias —aunque ya se postulaba desde antiguo en competencia con el corazónque el órgano de la mente es el cerebro? Permítaseme el atrevimiento de no valorar aquí los argumentos de aquellos que insisten una y otra vez en que la mente no existe o que es una pura apariencia, lo cual es propio - a mi parecer, como he justificado en otros textos- de posiciones evolucionistas radicales. A mi juicio, no valorarlos ahora, además de que contravienen la evidencia y llevan sobre ellos la carga de la prueba, tiene la función de ajustarme al tiempo que se me ha asignado y en el que, si lo hiciera, tendría también que valorar los argumentos de aquellos que afirman que el cuerpo no existe.

¿Existe la mente en el cerebro? No en el sentido del "cerebro en la cubeta" de H. Putnam. Hay que ver si el cerebro es capaz de explicar toda la actividad de la mente. En este sentido apelo, aunque habría otras instancias 
también a las que acudir, a las teorías de la cognición extendida: no es solo el cerebro, sino todo el cuerpo y el medio ambiente los que posibilitan el conocer. La mente no está "dentro" del cerebro sino que la cognición se abre a todo el ámbito de la realidad: la mente no está localizada en el cerebro, no está "dentro" de él. Si la mente existe no podemos referirnos a ella en términos de espacio localizándola en un lugar concreto: toda el alma está en todo el cuerpo y aún más allá del cuerpo.

Otra parte del argumento de Aristóteles guarda un fuerte sentido ontológico, aunque a primera vista pudiera parecer que no cuenta con la experiencia inmediata de que, como la facultad sensorial, la base fisiológica de la mente también se cansa de pensar. La mente tiene una base fisiológica cerebral que necesita recuperar energías a través del descanso y del sueño. Pero, y esto está bien visto, así como la facultad sensorial —el oído o la vista, por ejemplo- tiene un umbral crítico objetivo de percepción que no puede superar sin quedarse el sujeto sentiente sordo o ciego, el pensamiento, la actividad de la mente, no lo tiene: el pensar siempre puede conocer más e ir más allá de todo límite impuesto a priori. Ascender en el conocimiento concede más sentido a todo lo aprendido con anterioridad.

La tercera parte del argumento se cifra en la reflexividad: la mente puede inteligirse, puede conocer su naturaleza como objeto, aunque no pueda agotarse en la idea que se haga de sí misma: es un sujeto, es decir, trasciende toda objetividad. Esa es una característica muy especial que solo posee la mente: cuando el estómago se digiere a sí mismo implica la muerte del cuerpo; sin embargo, cuando la mente se intelige se da en ello su máxima plenitud, la expresión máxima de toda la ontología de lo finito. Una plenitud que supone, afirma Aristóteles de manera misteriosa para los que no nos movemos en el ámbito de los sabios, la pervivencia de la actividad tras la descomposición del cuerpo. 
Francisco Rodríguez Valls

\section{La dificultad de demostrar la separabilidad de la mente}

Ahí quedan los argumentos de Aristóteles, esa es su postura, pero están lejos de ofrecer una claridad meridiana que evite la discusión. Creo que revisten una gran dificultad, al menos para una inmensa mayoría que no nos movemos, como ya he dicho, en los círculos en los que estos temas se entienden como no problemáticos "apud sapientes tantum". De hecho llevamos dos mil quinientos años discutiendo esos textos. Argumentos sobre la separabilidad de la mente o sobre la inmortalidad del alma ha habido algunos, pero ninguno ha quedado exento de críticas. Hasta tal punto esa es una cuestión de hecho que, el 19 de diciembre del año 1513, el Papa León X publica la Bula Apostolici Regiminis que, además de condenar las doctrinas que afirmaban la mortalidad del alma humana, invita a los filósofos y sabios en general a buscar argumentos definitivos para demostrar lo contrario. Para el cristianismo la inmortalidad del alma es un dato revelado y debe — sospecho, aunque muy personalmente no estoy seguro— ser compatible con una demostración racional. Me encuentro en estos momentos en una situación grave puesto que debo prestar obediencia a una invitación papal que proviene de comienzos del siglo XVI, es decir, una vez que Tomás de Aquino esgrimió los suyos a favor y Pietro Pomponazzi los suyos en contra. La verdad sea dicha, la tarea me supera con mucho, como ha superado a tantos filósofos desde que el documento fuera promulgado. No obstante, no voy a desesperar de plantear algún camino por el que se vislumbre alguna luz, aunque sea para miradas miopes como la mía.

Por referirme al conocimiento contemporáneo, no creo que los intentos que la corriente general naturalista de la filosofía y de la ciencia ha hecho por explicar la naturaleza de la mente estén en condiciones de justificar su separabilidad. Más bien, desde puntos de vista tanto científicos como filosóficos, desde los Churchland, Damasio, Dennett, hasta Penrose, lo que se da son diferentes intentos de explicación de la conciencia que profundizan en su naturaleza material, pero no en su naturaleza espiritual y reflexiva en el sentido aristotélico del término que hemos visto hace unos momentos. 
Ya sea eliminar la conciencia directamente como un producto evolutivamente inútil o hacerla depender de un programa de investigación cuántico no algorítmico basado en el funcionamiento de los microtúbulos de tubulina, no dejan de formar parte del mismo bando. Esa tendencia general postularía que existe una explicación natural de la mente que, o la deja fuera con la misma realidad que la existencia de los elfos o de Santa Claus, o bien está en relación con una naturaleza material, sea esta de la naturaleza que sea.

Considero que lo mismo ocurre con las propuestas que hacen los partidarios de la inteligencia artificial respecto de la naturaleza de la mente. Es cierto que se acabaron ya los tiempos de concebirla como un simple ordenador más de funcionamiento en serie. En todo caso es un superordenador que funciona en paralelo y que es capaz de darse reglas y, por tanto, de autoprogramarse. Por un momento, consideremos el tema de la separabilidad de la mente no desde un punto de vista ontológico sino físico. Y, a mi parecer, eso es - en el fondo- lo que nos propone el subtítulo del simposio que celebramos: "¿puede la mente humana transferirse a un soporte no biológico?”, es decir, ¿puede el software en el que consiste la mente cambiar de un hardware basado en la química del carbono a otros basados, por ejemplo, en la del silicio? Y la respuesta que se me ocurre es que eso es necesario siempre y cuando la mente no sea separable y necesite un soporte nuevo para seguir viviendo una vez que las condiciones de su organismo biológico vayan a terminar.

Si la mente fuera separable y fuera espiritual tendría garantizada la vida eterna. ¿ंO, pregunto, no la tendría? Incluso desde ciertos puntos de vista religiosos cristianos, no digamos ya judíos, supone un problema qué ocurre entre el momento de la muerte y el de la resurrección de los cuerpos. ¿Tiene sentido un estado de alma separada en el que todos coinciden que resulta extraño para el alma misma -el alma separada no es el ser humano- y que, desde luego, no es el estado último de inmortalidad de lo humano? Solo con la resurrección de los cuerpos será posible el advenimiento de los Cielos Nuevos y de la Tierra Nueva en las que, eso sí, cada 
alma separada volverá a ocupar exactamente el cuerpo — glorioso y transfigurado- que ocupó antes de la muerte. No tenemos argumentos suficientemente fuertes como para que quede fuera de toda duda la inmortalidad o el estado de alma separada. Raramente los argumentos filosóficos tienen tal fuerza. Si buscamos la posibilidad de transferir la mente a un avatar no biológico es porque no tenemos nada claro que exista un alma inmortal separable y, sin embargo, lo que sí tenemos claro es que queremos vivir si puede ser en las mejores condiciones posibles: un avatar que no envejezca, que no enferme, que pueda aumentar sus conocimientos exponencialmente y, sobre todo, que se le permita la posibilidad de ser libre y feliz. Lo que buscamos en este simposio es, realmente, saber si existe la posibilidad o no de una segunda oportunidad en la que el ser humano haga las veces de su propio creador. A la manera del Doctor Frankenstein, lo que pretendemos es crear entidades, en este caso no biológicas, que nos permitan alcanzar el sueño de Prometeo que consiste en el bien para la humanidad, y especialmente para nosotros mismos como parte muy interesada de la humanidad, en una sociedad libre y sin conflictos.

\section{La inteligencia artificial, el mejoramiento humano y la mente como software. El problema del interfaz}

Como en algunas películas de ciencia ficción, se trata de hacer pasar el alma intelectiva de un cuerpo a otro que, en este caso, no es biológico sino sintético. Y hay que hacerlo apelando a la ciencia y no al conjuro ya que éste último se ha manifestado claramente inútil para resolver problemas de índole práctica material. El problema consistirá — hablemos en futuro- en que tendremos la tecnología suficiente como para crear un soporte no biológico con principio vital pero que no generaría mente - no la autogeneraría, o no queremos que la autogenere- y que habría que dársela. No es nuestra línea de razonamiento el ocuparnos entonces de la máquina sintética que evoluciona sola hasta hacerse humana, como otras series de ciencia ficción han planteado. Tendríamos que tener un ente sintético vivo 
y sin mente al que transferir una de un sujeto biológico natural que está agotando su existencia o que, sin más, lo desea porque la tecnología lo hace posible. Podría darse el caso de que ese conjunto de acciones se pusiera de moda y se convirtieran en algo así como querer cambiar de cuerpo como regalo de cumpleaños como ahora compramos, por ese motivo, un móvil a nuestros hijos. Sería parte del supuesto "mejoramiento" humano —y de la ética que acompaña al transhumanismo - realizar esta tarea. No se trataría de clonar otro ser de existencia vital y mentalmente autónoma, se trata de proporcionar otra base química a la vida intelectual que ya existe para que exista de mejor manera. Los argumentos que se esgrimieron en el curso de verano de Ávila de 2017 titulado "Humanos e Inhumanos", y que se encuentran recogidos en el número 10 de la revista Naturaleza y libertad, en los que se discute una ética transhumanista radical están ahí. No voy a retomarlos. Pero, en última instancia, ya que el transhumanismo nació de uno de los Huxley (Julian Sorell), establecer en el mundo feliz de otro Huxley (Aldous Leonard) que en lugar de soma exista una transferencia corporal que evite toda desgracia física y psicológica es lo que se persigue planteando estas cuestiones. En el fondo, lo que late bajo todos estos planteamientos es el miedo al dolor, a la vejez y a la muerte. Y el profundo y atávico deseo de los seres humanos de huir de ellos. Y queremos hacer de dioses recreando la creación al menos, y de momento, de aquello que más de cerca nos afecta y de lo que más nuestro nos hace: la interioridad mental.

Pero veo un problema y ese es el del interfaz que debe recoger la mente biológica natural para transferirla a la entidad no biológica y sintética que seguiría siendo, si se consigue la transferencia y según los planteamientos transhumanistas, el mismo individuo mentalmente hablando. ¿Se recoge la mente en una especie de chip que se implanta en el nuevo ser? ¿No sería, planteo, el mismo problema que el de la glándula pineal: llegar a la entidad menos material posible para ver si de esa forma lo inmaterial engancha con lo material? Veo que si la mente es material, no sería el mismo problema, ya que se trataría de recoger una actividad material en un sustrato material. Tendríamos que ver cómo se realiza técnicamente esa operación. Pero, si 
no lo es, si no fuera material, sí se trataría de una estrategia similar. Desde un punto de vista naturalista es un problema técnico. Desde el punto de vista humanista no tiene sentido plantearlo, como tampoco lo tiene la solución de Descartes habiendo pensado sus consecuencias durante tres siglos y medio después de que se expusiera.

Si la mente es material, transferirla de un sustrato biológico a otro no biológico es simplemente un problema técnico —el del interfaz- en el que deberán empeñarse los ingenieros y los científicos naturales en los próximos decenios. Y no harán mal en dirigir sus investigaciones en esa línea puesto que podrán hacer, esperemos, también lo esperaba Víctor Frankenstein, un gran bien a la especie humana.

Si la mente es separable y, por lo tanto, espiritual, creo que no cabe más posibilidad, aunque cueste lo indecible, que hacernos fuertes en nuestro destino mortal corporal y dejar que, en todo caso, los ángeles nos hablen.

\section{Conclusión}

Después de afirmado lo anterior tengo que entonar un mea culpa por no haber sabido hallar un argumento indiscutible sobre la materialidad o sobre la espiritualidad del alma y haberme limitado exclusivamente a plantear, de la manera más clara que he podido, el problema que nos reúne aquí. Tengo la esperanza y la creencia en su inmortalidad y en la naturaleza espiritual de la mente, pero me encuentro en ese bando que todavía, a pesar de que ha intentado con todas sus fuerzas buscar argumentos, sigue siendo apelado por la bula Apostolici Regiminis de León X. Es posible que la solución no esté en buscar argumentos por vías de la substancia material o espiritual sino de las acciones que derivan de lo que se supone material o espiritual y que, en consecuencia, afecten más a una metafísica de la relación o del don. Es por ese camino por el que va, por ejemplo, la obra de Josef Seifert Conocimiento de Dios por las vías de la razón y del amor (Encuentro. Madrid, 2013). O el que han emprendido los personalistas argumentando con la condición donal de 
la persona. Se trataría de pasar de la substancia a las acciones de la substancia, que reflejarían con claridad la naturaleza espiritual y trascendente de su condición.

Mi problema es que he bebido demasiado de las fuentes clásicas, especialmente de Aristóteles, y se me hace muy cuesta arriba hacer una filosofía sobre bases más débiles a priori — perdón por mis prejuicios— que la de la categoría de substancia, como puede ser la de relación. No voy a negarle mérito a los que emprenden ese camino, considero que tienen el espíritu de los pioneros que a mí, con mi edad, ya me va faltando y prefiero afianzarme en lo seguro. Aunque eso seguro no lleve a otro lado que a seguir pensando y repensando una y otra vez porque, como decía el Apóstol, es duro dar coces contra el aguijón.

Francisco Rodríguez Valls rvalls@us.es 